\title{
Detecção precoce do câncer de pele na atenção básica
}

\author{
Early detection of skin cancer in primary care
}

Detección precoz de cáncer de piel en atención primaria

Camila Baquieti Carminate ${ }^{1 *}$, Ágatha Barbosa Rocha1, Bárbara Poggiali Gomes ${ }^{1}$, Frederico Noboro Figueiredo Nakagawa1, Gustavo Lopes de Oliveira1', Júlia Fraga Vieira², Larissa Mariani Rezende Almeida ${ }^{1}$, Luísa Dias Toledo Ferreira ${ }^{1}$, Pedro Duarte Moreira Andrade ${ }^{1}$, Rafaela Almeida Silva1.

\section{RESUMO}

Objetivo: Analisar o papel da Atenção Básica na detecção precoce do câncer de pele, visando mostrar as principais estratégias utilizadas. Revisão bibliográfica: $O$ câncer de pele é a neoplasia que possui maior incidência no Brasil. A identificação do câncer em fase inicial, precoce, ou ainda de lesões pré-malignas, tais como queratoses actínicas e nevos melanocíticos displásicos, é um fator de bom prognostico devido ao tratamento precoce, com maiores chances de cura e menores sequelas cirúrgicas. Devido ao fato de a pele ser um órgão de fácil visualização, é importante orientar a população em relação aos sinais de alerta. Os médicos de família devem fazer o rastreio baseado no exame clínico visual da pele, incluindo a regra do ABCDE (assimetria da lesão, bordas irregulares, heterogeneidade de cor; diâmetro mais de $6 \mathrm{~mm}$ e evolução ao longo do tempo), pode-se realizar também exames auxiliares, como a dermatoscopia, exame confocal e mapeamento das lesões estudos feitos pelo dermatologista. Considerações finais: É importante orientar o paciente em reação da realização do autoexame, pelo menos uma vez no mês, para estar sempre atento às possíveis alterações.

Palavras-chave: Detecção precoce, Câncer de pele, Atenção básica.

\begin{abstract}
Objective: To analyze the role of Primary Care in the early detection of skin cancer, aiming to show the main strategies used. Bibliographic review: Skin cancer is the neoplasm that has the highest incidence in Brazil. The identification of cancer at an early, early stage, or even premalignant lesions, such as actinic keratoses and dysplastic melanocytic nevi, is a good prognostic factor due to early treatment, with greater chances of cure and fewer surgical sequelae. Due to the fact that the skin is an easily visualized organ, it is important to guide the population in relation to warning signs. Family physicians should perform the screening based on clinical visual examination of the skin, including the ABCDE rule (lesion asymmetry, irregular edges, color heterogeneity; diameter greater than $6 \mathrm{~mm}$ and evolution over time). auxiliary exams, such as dermoscopy, confocal exam and mapping of lesions, studies carried out by the dermatologist. Final considerations: It is important to guide the patient in reaction to the self-examination, at least once a month, to be always aware of possible changes.
\end{abstract}

Keywords: Early detection, Skin cancer, Primary care.

${ }^{1}$ Faculdade de Medicina Vale do Aço (UNIVAÇO), Ipatinga - MG.

*E-mail: camilacarminate@outlook.com

2 Pontifícia Universidade Católica de Minas Gerais (PUC Minas), Betim - MG.

SUBMETIDO EM: 8/2021

PUBLICADO EM: 9/2021 


\section{RESUMEN}

Objetivo: Analizar el papel de la Atención Primaria en la detección precoz del cáncer de piel, con el objetivo de mostrar las principales estrategias utilizadas. Revisión bibliográfica: El cáncer de piel es la neoplasia de mayor incidencia en Brasil. La identificación precoz, precoz o incluso de lesiones premalignas del cáncer, como las queratosis actínicas y los nevos melanocíticos displásicos, es un factor de buen pronóstico debido a un tratamiento precoz, con mayores posibilidades de curación y menos secuelas quirúrgicas. Debido a que la piel es un órgano de fácil visualización, es importante orientar a la población en relación a las señales de alerta. Los médicos de familia deben realizar el cribado en base al examen clínico visual de la piel, incluida la regla $A B C D E$ (asimetría de la lesión, bordes irregulares, heterogeneidad de color; diámetro mayor de $6 \mathrm{~mm}$ y evolución en el tiempo). Exámenes auxiliares, como dermatoscopia, examen confocal y mapeo de lesiones, estudios realizados por el dermatólogo. Consideraciones finales: Es importante orientar al paciente en la reacción al autoexamen, al menos una vez al mes, para estar siempre pendiente de posibles cambios.

Palabras clave: Detección precoz, Cáncer de piel, Atención primaria.

\section{INTRODUÇÃO}

O câncer é considerado uma patologia que possui uma etiologia multifatorial, sendo resultante de mutações genéticas, fatores ambientais e do estilo de vida do paciente (POPIM RC, et al., 2008). Sendo assim, existe muitos outros tipos de doenças malignas que têm o crescimento desordenado de células, que acabam invadindo os tecidos adjacentes ou órgãos a distância causando metástase (INSTITUTO NACIONAL DE CÂNCER, 2020).

Uma das causas do surgimento do Câncer de Pele pode estar relacionada diretamente com o fator p53 mutado, sendo o p53 um gene supressor tumoral que é possível de ser encontrado em muitos tumores malignos e benignos, cuja sua principal função é manter as células em estado de repouso, levando a ter um dano no DNA da célula (POPIM RC, et al., 2008). Assim como, os raios UV constituem um principal fator de risco evitável para a doença, eles atuam no DNA celular, causando mutações e na pele através de processo inflamatório crônico, levando ao desenvolvimento da neoplasia (WOLFF K, et al., 2014).

O câncer de pele é considerado a neoplasia com maior incidência no Brasil (CASTILHO IG, et al., 2010). Com base em dados estatísticos dos últimos anos, é possível concluir que o número de casos novos de câncer de pele não melanoma esperados, entre os anos de 2020-2022, será em média 83.770 nos homens e 93.160 nas mulheres. Sendo o câncer de pele não melanoma, mais especificamente em homens, mais incidente nas Regiões Sul, Centro-Oeste e Sudeste, podendo ser estimado cerca de 85,55/100 mil casos somente na região sudeste. No que diz respeito às mulheres, é mais incidente em todas as Regiões brasileiras, sendo estimado cerca de 100,85/100 mil casos na região Sudeste. Em relação ao câncer de pele melanoma, o número de casos novos estimados é de 4.200 em homens e 4.250 em mulheres (INSTITUTO NACIONAL DE CÂNCER, 2019).

Alguns fatores podem ser atribuídos para o risco do desenvolvimento dessa neoplasia, tais como: cor da pele, horário e tempo de exposição ao sol, residência em país tropical e uso de imunossupressão crônica. É possível compreender que o câncer da pele é dividido em dois tipos, sendo eles não melanoma (Carcinoma Basocelular ou Carcinoma Epidermoide) e melanoma (INSTITUTO NACIONAL DE CÂNCER, 2014). O câncer de pele apresenta-se como uma das neoplasias com melhor prognóstico se diagnosticado precocemente (SELLA F, 2017).

As evidências são insuficientes para se avaliar a importância para a recomendação do exame de toda a extensão pele por um médico de atenção primária, com o intuito do diagnóstico precoce (MINISTÉRIO DA SAÚDE, 2010). A identificação do câncer em fase inicial, precoce, ou ainda de lesões pré-malignas, tais como queratoses actínicas e nevos melanocíticos displásicos, é um fator de bom prognostico devido ao tratamento 
precoce, com maiores chances de cura e menores sequelas cirúrgicas (INSTITUTO NACIONAL DE CÂNCER, 2016). Devido ao fato de a pele ser um órgão de fácil visualização, é importante orientar a população em relação aos sinais de alerta (MINISTÉRIO DA SAÚDE, 2010).

Portanto, é de estrema relevância que os profissionais de saúde da unidade básica realizarem o diagnóstico precoce do câncer de pele através da detecção de sinais e sintomas, sendo elas: manchas que coçam, doem, sangram ou descamam; feridas que não cicatrizam em quatro semanas; sinais que mudam de cor, textura, tamanho, espessura ou contornos; elevações ou nódulos da pele circunscritos e adquiridos que aumentam de tamanho e tenham a aparência perolada, translúcida, avermelhada ou escura (MINISTÉRIO DA SAÚDE, 2010).

Vale ressaltar que as estratégias de diagnóstico precoce citadas acima, devem fazer parte da rotina das equipes de Saúde da Família e Comunidade (MINISTÉRIO DA SAÚDE, 2010). Os médicos de família devem fazer o rastreio baseado no exame clínico visual da pele, incluindo a regra do ABCDE (assimetria da lesão, bordas irregulares, heterogeneidade de cor; diâmetro mais de $6 \mathrm{~mm}$ e evolução ao longo do tempo), pode-se realizar também exames auxiliares, como a dermatoscopia, exame confocal e mapeamento das lesões estudos feitos pelo dermatologista (NAPOLES RLCL, 2018).

O objetivo do presente artigo é analisar o papel da Atenção Básica na detecção precoce do câncer de pele, visando identificar as melhores estratégias para as exigências advindas do cenário da saúde atual. Assim como, sugerir estratégias que possam ser desempenhadas pela Saúde as Família neste contexto de prevenção.

\section{REVISÃO BIBLIOGRAFICA}

Câncer de pele é considerado o câncer mais comum no mundo e no Brasil, sendo o melanoma, carcinoma basocelular, originado de células não queratinizantes, e o carcinoma espinocelular, derivados dos ceratinócitos, os tipos mais frequentes da doença (INSTITUTO NACIONAL DE CÂNCER, 2016). Esses dois tipos de tumores não melanoma possuem formas diferentes que os torna possíveis de diferenciar no exame clinico e histopatológico, entretanto ambos são parecidos em relação ao prognóstico, apresentando baixa letalidade (COSTA CS, 2012).

O câncer de pele não melanoma (CPNM) corresponde a 90\% de todos os tipos de canceres de pele, com sua incidência vem aumentado e principalmente numa faixa etária mais jovem, sendo assim, a prevenção e o diagnóstico precoce têm um substancial significado na saúde pública (ZINK BS, 2014). Sendo o melanoma, entre as neoplasias de pele, o câncer de pior prognóstico (MINISTÉRIO DA SAÚDE, 2013).

A pele é o maior órgão do corpo humano, dessa forma, ele reveste, protegendo o organismo e interagindo com o meio externo (BARDINI G, et al, 2012). Sendo ela, composta de três grandes camadas de tecidos: epiderme, a superior; a intermediária (derme); e a hipoderme, profunda (RIVITTI EA, 2014). A pele funciona como uma proteção do corpo contra o calor, luz, infecções e é responsável por regular a temperatura do corpo e auxilia reservas de água, vitamina $\mathrm{D}$ e gordura (BARDINI G, et al, 2012). Portanto, é um órgão que pode executar diversas funções, como: barreira, proteção imunológica, termorregulação e secreção (RIVITTI EA, 2014).

A melanina, presente na pele, tem um importante papel na proteção da pele contra a radiação ultravioleta proveniente do sol pela absorção da energia irradiante, não só absorvendo como também difundindo a radiação (RIVITTI EA, 2014). Mesmo assim, a pele é suscetível a ser atingida por fenômenos patogênicos capazes de causar alterações microscópicas fundamentais que macroscopicamente transformam-se em lesões elementares, dentre elas as neoplasias (BARDINI G, et al, 2012).

O câncer decorre do crescimento anormal e descontrolado das células que compõem as camadas pele, portanto, dependendo da camada afetada é possível definir e diferenciar dos diferentes tipos de câncer de pele (SILVA ALA, et al, 2015). Esse crescimento anormal de células, fazem com elas se dispõem em camadas, constituindo em diferentes tipos de células (MOREIRA APA, et al, 2015). 
O câncer de pele é o mais comum e possível de ser prevenível dentre todos os tipos de cânceres (BARDINI $G$, et al, 2012). O câncer de pele é uma neoplasia ocupacional, que tem ocorrido devido à exposição exagerada a produtos químicos cancerígenos, como arsênico (MOREIRA APA, et al, 2015).

Câncer de pele melanoma tem baixa incidência e alta letalidade, sendo que aparece raramente, mas com repercussões graves para os pacientes, podendo levar à morte (COSTA CS, 2012). Existe uma diferença na predominância do melanoma entre os gêneros, sendo na mulher mais comumente encontrada nas pernas ou cabeça e nos homens mais comumente encontrada no tronco. Esse fato ocorre devido as variações de comportamento entre os gêneros, como por exemplo as profissões, vestuários e exposição ao sol (ALMEIDA FT, et al., 2019).

Os Carcinomas Basocelulares costumam apresentar-se como placas eritematosas de crescimento lento, sendo mais invasivos e apresentam um estágio tardio devido á aparência pálida e mal demarcada. Os Carcinomas Espinocelulares apresentam-se como úlceras ou lesões endurecidas, que evoluem ao longo de vários meses com involução espontânea (GRIFFIN LL, et al., 2016). O melanoma é um tumor de origem neuroectodérmica, com isso os melanócitos são as células que derivam da crista neural, migrando na embriogênese por toda a epiderme, sendo encontrados principalmente em na camada basal (MATHEUS LGM e VERRI BHMA, 2015).

As lesões do câncer de pele têm crescimento lento e as metástases são raras, mas a invasão local e a destruição das estruturas vizinhas podem ocorrer se não tratadas (GRIFFIN LL, et al., 2016). O risco de metástase é maior para lesões que envolvem o couro cabeludo, testa, orelhas, nariz e lábios. Lesões indiferenciadas com mais de $6 \mathrm{~mm}$ de espessura que passaram a invadir estruturas mais profundas, incluindo a musculatura, pericôndrio ou periósteo, também apresentam um risco aumentado de metástase (NAHHAS AF, et al., 2017).

A principal causa de câncer de pele é a grande exposição à Radiação Ultravioleta (UV) natural proveniente do sol, sendo o espinocelular comumente associada a essa exposição crônica e durante a infância tem associação com os basocelulares e melanomas (PCRJ, 2016). Os raios UV causam alterações como: danificação no Ácido Desoxirribonucleico (DNA) das células da pele; produção de moléculas ativadas por oxigênio, resultando no dano ao DNA e estruturas moleculares; bloqueio da imunossupressão anticâncer das defesas naturais do corpo; podendo ter relação com a modificação dos genes p53 e patch (PTCH1), sendo eles os genes supressores da carcinogênese. Assim como, leva a uma inflamação da pele com aumento da produção de prostaglandinas, induzindo a síntese de ciclooxigenase 2 (COX-2) (ZINK BS, 2014).

A radiação ultravioleta é classificada em: Radiação Ultravioleta C (UVC), com o menor comprimento de onda e que possui efeitos carcinogênicos e mutagênicos; Radiação Ultravioleta B (UVB), que causam queimaduras, bolhas, lesões e câncer de pele; UVA que tem o maior comprimento de onda, sendo assim é a que causa mais danos por penetrar mais profundamente a derme e ocasionar a geração de radicais livre, ocasionando a produção de melanogênese (SILVA ALA, et al, 2015). Os raios da Radiação Ultravioleta A (UVA) são capazes de manter a mesma intensidade durante o ano todo, produzem alterações como manchas, fotoenvelhecimento e câncer. Os raios UVB possuem ondas medias com menor poder de penetração na pele, porém tem um potencial nocivo relevante, causando danos principalmente em nível de DNA celular (MOREIRA APA, et al, 2015).

Com o passar dos anos a radiação vem aumentando cada vez mais, o que leva a compreender que o uso de protetores solares é essencial para prevenção de doenças de pele, tais como o câncer de pele (SILVA ALA, et al, 2015). O fato de a radiação ultravioleta estar aumentando, está diretamente relacionada ao afinamento da camada de oxônio (MOREIRA APA, et al, 2015).

Alguns fatores que influenciam o aumento da chance de câncer de pele incluem: pele, olhos e cabelos claros; história familiar; sistema imune debilitado; imunossupressores. Alguns fatores ambientais e ocupacionais podem influenciar, como a exposição a fuligens, arsênico e seus compostos, alcatrão de carvão, óleos minerais e óleos de xisto (COSTA CS, 2012). Algumas outras condições podem aumentar o risco de desenvolver CPNM, sendo eles: exposição aos raios ionizantes; fototerapia com UVA; úlcera venosa crônica; 
osteomielite crônica; portadores de diversas dermatoses (ZINK BS, 2014). A etnia também pode ser inclusa como um dos fatores de suscetibilidade para a incidência de câncer de pele (ZINK BS, 2014).

As manifestações cutâneas causadas pelo excesso de exposição ao sol, apresentam um aspecto evolutivo, sendo ela: queimadura, espessamento da pele, manchas hipercrônicas, rugas finas, rugas profundas, ceratose actínica e câncer de pele (BARDINI G, et al, 2012).

Portanto, câncer de pele pode ser prevenido, sendo importante que a população seja engajada a adotar comportamentos de proteção solar (ROSSI DS, et al., 2018). Estratégias de prevenção estão relacionadas a encorajar mudanças comportamentais para reduzir o risco de câncer de pele subsequente e para aumentar a detecção precoce (APALLA Z, et al., 2017). O alvo dessas prevenções devem ser especialmente as crianças, visto que elas se expõem cerca de três vezes mais que os adultos ao sole o risco de desenvolvimento de câncer está relacionado com a exposição cumulativa na infância e adolescência (LAGES $\mathrm{RB}$, et al., 2012).

É de extrema importância que os profissionais de saúde, especialmente os médicos de família e comunidade orientem os pacientes a adotarem medidas de prevenção do cancer de pele. Algumas das principais recomendações para prevenção são evitar exposição ao sol em horários que os raios são mais intensos, uso de óculos de sol, roupas que protegem o corpo, chapéu com abas largas, sombrinhas, guarda sol e protetores labiais (PCRJ, 2016). Assim como, uso de filtro solar com fator de proteção solar (FPS) 15 ou mais, mesmo não existindo evidencias suficientes para sugerir que isolada previne o câncer de pele, sendo que vale ressaltar que mesmo aplicada de forma rotineira, não é capaz de levar a deficiência de vitamina $D$. (COSTA CS, 2012).

Utilização de companhas através da mídia para a educação pública com a finalidade de alcançar a modificação comportamental é essencial e econômica (APALLA Z, et al., 2017). Realização periódica de atividades educativas na comunidade referentes aos fatores de risco e de proteção, bem como, aos sinais para detecção precoce do câncer da pele através do autoexame (PCRJ, 2016).

A maioria das neoplasias malignas cutâneas são assintomáticas e não constituem o motivo da consulta, mostrando a importância do exame clinico detalhado (LAGES RB, et. al., 2012). Sendo assim, o diagnóstico precoce e acurado de lesões iniciais, implica em menores chances de deformidades cicatriciais e prejuízo funcional em decorrência do tratamento cirúrgico ou a radioterapia (COSTA CS, 2012).

Para a realização da detecção precoce do câncer de pele, algumas estratégias são utilizadas, como aplicação de exames individuais saudáveis, sem sinais e sintomas da doença, com o objetivo de detectar a doença em fase pré-clínica. Dessa forma, médicos da atenção básica devem estar alertas para lesões de pele com características malignas durante o exame físico e encaminhar para biopsia, sendo uma das estratégias utilizadas a regra do $\mathrm{ABCDE}$, que se baseia na observação de características de nevos, como a assimetria, bordas, cor, diâmetro e evolução (PCRJ, 2016). Entretanto, os critérios A e C podem ser mais favoráveis ao diagnóstico de melanoma (ROSSI DS, et al., 2018).

Existe três níveis de prevenção: primaria, que está relacionada a prevenção de riscos de determinada enfermidade; a secundaria, que se relaciona no diagnóstico precoce; e a terciaria que se refere a prevenção de deformidades, recidivas e até mesmo a morte (BOMFIM SS, et al., 2018). Dentre os princípios de detecção precoce do câncer de pele, o enfermeiro da unidade de saúde deve orientar os pacientes a se protegerem de superfícies que são refletoras (areia, neve, concreto e água), usar hidratantes corporais após exposição ao sol e evitar substancias que aumentam a sensibilidade em contato ao sol, como laranja e limão (MOREIRA APA, et al, 2015).

A prevenção secundaria está relacionada com o rastreio e o diagnóstico precoce junto com o aconselhamento da população para que desenvolvam práticas de atitudes relacionadas a prevenção no dia a dia deles (BARDINI G, et al., 2012). A detecção precoce auxilia na melhora do prognostico auxiliando a redução da neoplasia, sendo assim, identificando fenótipos de risco pode favorecer ações de saúde pública que visam à prevenção primaria e secundaria (BOMFIM SS, et al., 2018). Além disso, são fundamentais na redução da morbimortalidade e seu impacto na saúde pública (MOREIRA APA, et al., 2015). 
Assim como, a autopercepção do próprio corpo, como a pratica do autoexame da pele, e suas alterações não costumam fazer parte do cotidiano dos brasileiros, o que influencia no diagnóstico precoce e no prognostico das neoplasias cutâneas (RIBEIRO JPJ, et al., 2020). Dessa forma, a atuação do enfermeiro irá estar voltada para redução da exposição da população a fatores de risco, com o foco em diminuir a ocorrência dessa neoplasia por meio da promoção da saúde, através de proteção especifica e reorientações de atitudes e hábitos (MOREIRA APA, et al., 2015).

A dermatoscopia é um método não invasivo, utilizado pelo dermatologista, que permite analisar mais detalhadamente e com uma melhor visualização das eventuais lesões cutâneas, facilitando a diferenciação de outras doenças clinicamente confundidas com melanoma (MINISTÉRIO DA SAÚDE, 2013). Sendo assim, nevos que apresentam alteração de pigmentação, assimétrica, inflamação, ulceração e sangramento, possuem maior risco de malignização (PCRJ, 2016).

O uso da biopsia é indicado para confirmação diagnostica de uma lesão suspeita de câncer de pele (PCRJ, 2016). A biópsia deve ter como alvo a profundidade da derme reticular profunda se houver preocupação com invasão local, embora as recomendações para margens profundas durante a excisão não sejam especificadas (NAHHAS AF, et al., 2017). Biópsia excisional da lesão deve ser realizada em pacientes com lesões suspeitas de melanoma, sendo confirmado por exame histopatológico. Biópsia incisional é utilizada caso a excisional não possa ser realizada devido ao tamanho ou à localização da lesão (MINISTÉRIO DA SAÚDE, 2013).

No exame anatomopatológico, devem estar descritos os seguintes achados, como: diagnóstico e subtipo de melanoma, margens, crescimento radial ou vertical associado, espessura tumoral, presença de ulceração, invasão vascular ou perineural, regressão, infiltrado inflamatório e índice mitótico (MINISTÉRIO DA SAÚDE, 2013). O uso de tomografia de coerência pode ser útil para melhorar a precisão do diagnóstico em alguns cânceres de pele (APALLA Z, et al., 2017).

A escolha do tratamento irá se basear no comportamento biológico do tumor e com a presença de pelo menos um dos fatores de risco, como: diâmetro maior que $20 \mathrm{~mm}$ no tronco ou em extremidades; diâmetro maior que $10 \mathrm{~mm}$ em cabeça ou pescoço; diâmetro maior que $6 \mathrm{~mm}$ em genitália, mãos ou pés; recidiva; imunossupressão; tipo histológico; e acometimento perineural (ZINK BS, 2014). Por via de regra, o tratamento do câncer de pele não melanoma consiste em uma cirurgia para retirada da lesão e em casos avançados ou câncer de pele melanoma, o tratamento varia com o tamanho e estadiamento do tumor, sendo indicadas cirurgia, radioterapia e quimioterapia (PCRJ, 2016). A radioterapia é constantemente utilizada para tratamento paliativo, visto que não se utiliza para tratamento devido ao tumor ser resistente (MINISTERIO DA SAÚDE, 2013).

Terapia Fotodinâmica Tópica (TFD), onde utiliza-se um agente fotossensibilizante, metil aminolevulinato ou ácido 5-aminolevulínico, ativado por uma fonte de luz, que irá produzir porfirinas fotoativas em queratinócitos malignos e resultara na liberação de espécies reativas de oxigênio e formação de radicais livres (GRIFFIN LL, et al., 2016). O uso de alfa-interferona (a-IFN) tem mostrado resultados promissores, mostrando melhora significativa no que tange a sobrevida do paciente livre de recidiva. Entretanto, o uso do tratamento com IFN tem impacto negativo na qualidade de vida, causando efeitos colaterais que acabam limitando seu uso prolongado, principalmente com doses mais altas (MINISTÉRIO DA SAÚDE, 2013).

\section{CONSIDERAÇÕES FINAIS}

O câncer de pele tem apresentado aumento em sua incidência nos últimos anos. Sendo assim, é importante orientar o paciente em relação da realização do autoexame, pelo menos uma vez no mês, para estar sempre atento às possíveis alterações. O autoexame deve ser realizado seguindo as instruções: examinar a parte anterior, posterior e lateral do corpo inteiro diante de um espelho, com os braços levantados; olhar parte externa e interna dos antebraços, braços, mãos, pernas e pés, incluindo plantas dos pés e os espaços entre os dedos; levantar o cabelo para examinar o pescoço e o couro cabeludo; e verificar a área genital e nádegas. Portanto, os Médicos de Família devem fazer um exame físico minucioso no paciente com suspeita, utilizando a regra do $A B C D E$ ou, caso preciso, dermatoscopia. 


\section{REFERÊNCIAS}

1. ALMEIDA FT, et al. Melanoma Maligno Cutâneo: Estudo Retrospectivo de Sete Anos (2011-2017). Revista SPDV, 2019; 77(2): 1-4.

2. APALLA Z, et al. Câncer de pele: epidemiologia, carga de doença, fisiopatologia, diagnóstico e abordagens terapêuticas. Dermatol Ther (Heidelb), 2017; 7(1): 5-19.

3. APALLA Z, et al. Tendencias Epidemiológicas no Câncer de Pele. Dermatol Pract Concept., 2017; 7(2): 1-6.

4. BARDINI G, et al. Evaluation of knowledge and habits of dermatological patients with regard to skin câncer. Arq. Catarin. Med. 2012; 41(2): 56-63.

5. BOMFIM SS, et al. Meta Skin Cancer: Knowing and Preventing Population. Rev. Cient. Sena Aires, $2018 ; 7(3): 255-9$.

6. CASTILHO IG, et al. Fotoexposição e fatores de risco para câncer da pele: uma avaliação de hábitos e conhecimentos entre estudantes Universitários. An Bras Dermatol., 2010; 85(2): 173-8.

7. COSTA CS. Epidemiologia do Câncer de Pele no Brasil e evidências sobre sua prevenção. Diagn Tratamento, 2012;17(4):206-8.

8. GRIFFIN LL, et al. Cancer de Pele Não Melanoma. Clin Med (Lond), 2016; 16 (1): $62-65$.

9. INSTITUTO NACIONAL DE CÂNCER. 2014. In: Câncer de Pele não melanoma. Disponível em: <https://www.inca.gov.br/tipos-de-cancer/cancer-de-pele-nao-melanoma>. Acessado em 02 Setembro 2021.

10. INSTITUTO NACIONAL DE CÂNCER. 2016. In: Detecção Precoce - Boletim ano 7, n. 3. Disponível em: <https://www.inca.gov.br/sites/ufu.sti.inca.local/files/media/document/informativo-deteccao-precoce-3-2016.pdf>. Acessado em 02 Setembro 2021.

11. INSTITUTO NACIONAL DE CÂNCER. 2019. In: Estimativa 2020: incidência de câncer no Brasil. Disponível em: <https://www.inca.gov.br/publicacoes/livros/estimativa-2020-incidencia-de-cancer-no-brasil>. Acessado em 02 Setembro 2021.

12. INSTITUTO NACIONAL DE CÂNCER. 2020. In: O que é Câncer?. Disponível em: <https://www.inca.gov.br/o-que-ecancer>. Acessado em 02 Setembro 2021.

13. LAGES RB, et. al. Detecção Precoce do Câncer de Pele: Experiencia de Campanha de Prevenção no Piauí-Brasil. Rev Bras Promoç Saúde, 2012; 25(2): 221-227.

14. MATHEUS LGM, VERRI BHM. A. Aspectos Epidemiológicos do Melanoma Cutâneo. Revista Ciência e Estudos Acadêmicos de Medicina, 2015; 3(1): 10-24.

15. MINISTÉRIO DA SAÚDE. 2010. In: Departamento de Atenção Básica: Rastreamento. Disponível em: $<$ https://bvsms.saude.gov.br/bvs/publicacoes/caderno_atencao_primaria_29_rastreamento.pdf>. Acessado em 02 Setembro 2021.

16. MINISTÉRIO DA SAÚDE. 2013. In: Diretrizes diagnósticas e terapêuticas do melanoma maligno cutâneo. Disponível em: <https://bvsms.saude.gov.br/bvs/saudelegis/sas/2013/prt0357_08_04_2013.html>. Acessado em 02 Setembro 2021.

17. MOREIRA APA, et al. Non-Melanoma Skin Cancer and Occupational Risk of Outdoor Workers: Integrative Review. Rev enferm UFPE on line., 2015; 9(12):1310-9.

18. NAHHAS AF, et al. Uma revisão das Diretrizes Globais sobre Margens Cirurgicas para Câncer de Pele Não Melanoma. J Clin Aesthet Dermatol., 2017;10(4):37-46.

19. NAPOLES RLCL. Plano de intervenção para prevenção e rastreamento de câncer de pele no Município de Botuverá, Santa Catarina. Dissertação UFSC, Florianópolis, 2018; 27p.

20. POPIM RC, et al. Câncer de pele: uso de medidas preventivas e perfil demográfico de um grupo de risco na cidade de Botucatu. Rev. Ciênc. Saúde Coletiva,2008; 13(4): 1331-1336.

21. PREFEITURA DA CIDADE DO RIO DE JANEIRO. Secretaria Municipal de Saúde (SMS). Superintendência de Atenção Primária. Coleção Guia de Referência Rápida: Câncer da pele identificação e conduta. Versão Profissional, Série F. Comunicação e Educação em Saúde. 1aㅗ edição. Riode Janeiro, 2013. Disponível em: http://www.rio.rj.gov.br/dlstatic/10112/6552790/4179802/GuiaPele.pdf. Acessado em 02 Setembro 2021.

22. RIBEIRO JPJ, et al. Action against skin cancer in a city with high ultraviolet index. Rev Bras Promoç Saúde, 2020; 33:10695.

23. RIVITTI EA. Manual de Dermatologia Clínica de Sampaio e Rivitti. Artes Médicas. 2nd ed. São Paulo: 2014, 748p.

24. ROSSI DS, et al. Prevenção e Detecção Precoce do Câncer de Pele. Acta méd, 2018; 39(2): $327-334$.

25. SELLA F. Plano de intervenção para prevenção e diagnóstico precoce de câncer de pele no Distrito Juvêncio, no município de Saudades-SC. Dissertação Departamento de Saúde Pública - Universidade Federal de Santa Catarina Florianópolis, 2017; 23p.

26. SILVA ALA, et al. A importância no uso de protetores solares na prevenção do fotoenvelhecimento e câncer de pele. Revista Interfaces: Saúde, Humanas e Tecnologia, 2015;3(1): 1-7.

27. SILVA WSC, MENDONÇA PBS. Intervenções de enfermagem na atenção básica quanto á prevenção ao câncer de pele não melanoma em idosos. VI Congresso Internacional de Envelhecimento Humano. 2019.

28. WOLFF K, et al. Dermatologia de Fitzpatrick. Porto Alegre: AMGH, 2014.

29. ZINK BS. Câncer de pele: a importância do seu diagnóstico, tratamento e prevenção. Revista HUPE, 2014; 13(1):7683. 